\title{
Automatic segmentation of spinal cord injury using coarse- to-fine strategy
}

\author{
Jiali Cui, Yubo $\mathrm{Ma}^{\mathrm{a}}$, Wei Xie, Yiding Wang and Ruiming Jia \\ College of Information Engineering, North China University of Technology, P.R.China, 100144
}

\begin{abstract}
Medical image segmentation is a key issue in the field of medical image processing and analysis. Segmentation of spinal cord injury draws interests of researchers recently. To eliminate human computer interaction in segmentation of spinal cord injury, an automatic segmentation of spinal cord injury is proposed. A self-adaptive non-edge information smoothing algorithm was presented to realize filtering processing of spinal cord injury (SCI) images. The first time to use Deformable Parts Model (DPM) algorithm on the positioning detection of spine in an SCI image. A large number of non-scar information noises were removed and the detection range of scars was further reduced. Based on Coarse-to-fine strategy, a fully-automated spinal cord scar injury segmentation algorithm is put forward. The experimental results demonstrated the effectiveness of this algorithm.
\end{abstract}

Keywords: medical image segmentation; automatic segmentation; spinal cord injury; coarseto-fine strategy.

\section{Introduction}

Medical image segmentation is aimed at segmenting the portion with particularized implicature from the medical images and extracting relevant characteristics. It can provide doctors reliable basis for clinical diagnosis and pathology research [1], [12-15].

With the consistent increasing of the occurrence rate of traffic accidents, the athletic injuries and mining accidents, the morbidity of SCI (see Figure.1) has been increasing year by year. According to the statistical data, the morbidity rate of SCI is 233-755/one million around the world. The annual morbidity is 10.4-83/one million. The permanent disability caused by SCI will lead to heavy burden for the families and the patients. It will seriously affect the quality of life of the patients. The astrocyte, microglial cell, oligodendrocyte and the extracellular matrix secreted after SCI will form glial scar (see Figure.5 and 8).

Generally, it is believed that early reactive colloid has certain protection effects on the nerve. It is conducive to survival and regeneration of nerve cell. Obvious glial scar will form one month after SCI. Researches showed that the damaged neuraxis axon stop growing access to the glial scar, as results in disability in movement.

\footnotetext{
a Corresponding author : mayubo1205@sina.com
} 


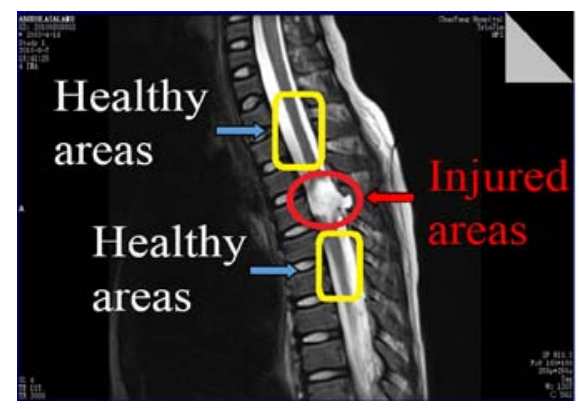

Figure 1. Spinal cord injury image

Segmentation of SCI is helpful for medical operation, so it is of vital importance to the rehabilitation of the patients [2]. The term segmentation means locating the size and location of spinal cord scar accurately. Thus, segmentation is also called as scar segmentation. Traditional medical image segmentation technologies can be categorized into two groups, namely region growing algorithm [3-4] and Snake algorithm [5-6]. These methods have their own advantages with certain shortcomings. And most of medical segmentation algorithms need manual intervention to select the initial position. Although human-computer interaction (HCI) can locate and segment regions of interest (ROI) based on the experience of physicians on the status of patients, it will significantly increase the workload of doctor diagnosis. Thus this paper's target is to fulfill an automatic segmentation of SCI. Since the injured areas and injury degree of patients with SCI are different, the location and size of scar are not fixed either. It is very difficult to perform direct and accurate scar location; therefore, a coarse-to-fine strategy is used.

\section{Segmentation of $\mathrm{SCl}$}

Firstly, in view of the characteristics of MRI and its noise problems, a self-adaptive non-edge information filtering smooth algorithm is put forward to filter SCI images. Secondly, to locate the SCI automatically and precisely, a coarse-to-fine strategy is designed and described as follows. The proposed method includes two main procedures, i.e. raw and precise localization. Raw localization combines DPM to decide which MRI slice is with spine and the initial scope of SCI. Precise localization gradually narrows the detection range using fuzzy c-means (FCM) algorithm and the boundary information. Finally it segments SCI using the region-growing algorithm effectively. The main flow chart of this paper is illustrated in Figure 2.

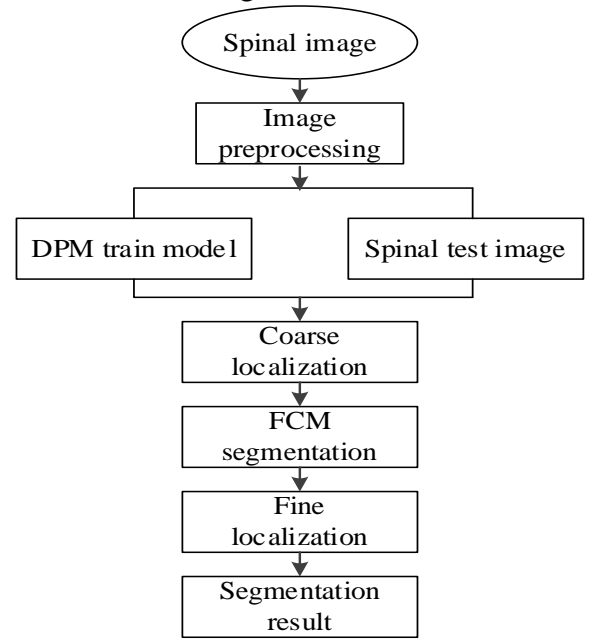

Figure 2. Main flow chart of the proposed method 


\subsection{Self-adaptive non-edge information smoothing algorithm}

Similar to traditional image processing, in any two-dimensional gray image, each pixel point was likely to be either a noise factor or an image pixel. As far as a medical image was concerned, most of the noises were generated in current pulses. Impulse noises were similar to salt \& pepper noises in traditional image noises and belonged to isolated noise points. Due to a great discrepancy between isolated noises and neighborhood pixels, all isolated noises in an image were determined with distance template method. Templates are shown in Figure 3.

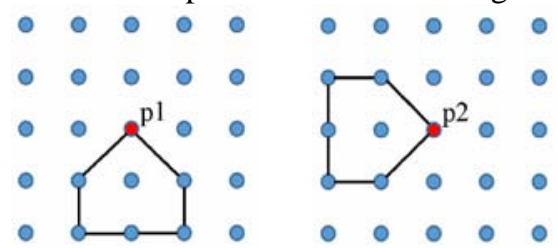

(a). Horizontal and vertical direction templates

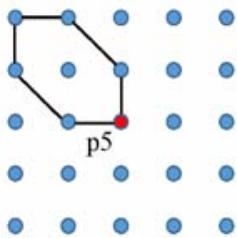

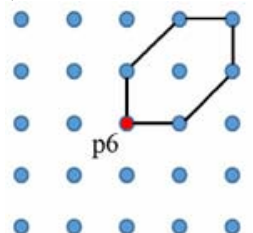

(b). 45 Degrees direction templates
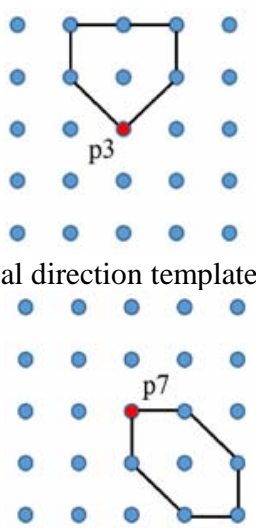
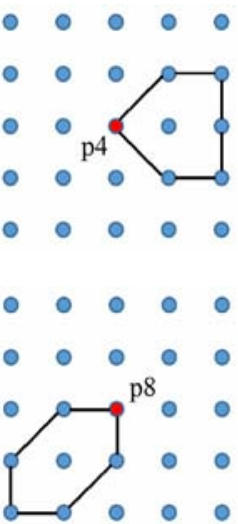

Figure 3. Filter template

\subsection{Raw localization using deformable part model}

Generally there are three sequences for MRI image of patients with SCI, namely axial, sagittal and coronal view and there are variable slices in each view. The relationship between connected slices denotes diversified patients' information. Thoracic vertebra cord injury of T2 weighted coronal images is shown in Figure 5 and 8, where SCI can be seen in selected slices. The procedure of raw localization decides slice index(which includes spine) and the rectangle which surrounds the spine(see Figure 5(d)).

DPM is adopted for raw spine localization in medical images because excellent performance has been acquired in PASCAL VOC recognition competition [7-9] since it is proposed by Felzenszwalb. DPM is an object detection model based on sliding windows.

The variable component model constitutes by a root filter, several component filters and a series of deformation parameters. These deformation parameters describe the deviation degree of actual position of the components comparatively to training location. While at matching, a certain size was given to the root filter of DPM. The moving window is adopted for all the locations of the image as well as all pyramid layers detection. The matching degree is measured through detection score. The test score is the sum of root filter score and filter components, minus the deformation cost. When the score of detection window is higher than the threshold, it indicates the objects tested, otherwise not. Set $\varphi(x, y)$ as the corresponding image characteristics of the detection window. $x$ marks the location of the detection window; $z$ marks the location of the component. Thus the resulting score of the image can be calculated through the following formula. $\beta$ is the parameter vector of the model. It can be obtained during the training phase of the classifier.

$$
\operatorname{score}(x)=\max _{z \in Z(x)} \beta \cdot \varphi(x, Z)
$$

During the training phase, the latent support vector machine (SVM) can be used. The training objective function is. 


$$
L_{D}(\beta)=\frac{1}{2}\|\beta\|^{2}+C \sum_{i=1}^{n} \max \left(0,1-y_{i} f_{\beta}\left(x_{i}\right)\right)
$$

Whereas in the formula, $x_{i}$ as the input image, $y_{i}$ is the flag value of the sample. The positive sample is marked as 1 and the negative sample is marked as -1 ; C is the constant to be used to control the degree of punishment to wrong samples. This paper uses 600 positive samples(images with spinal information) and 1200 negative samples(images without spinal information) as the training dataset of DPM model. The visualization of trained spine model is presented in Figure 4(see reference 7 for more information).
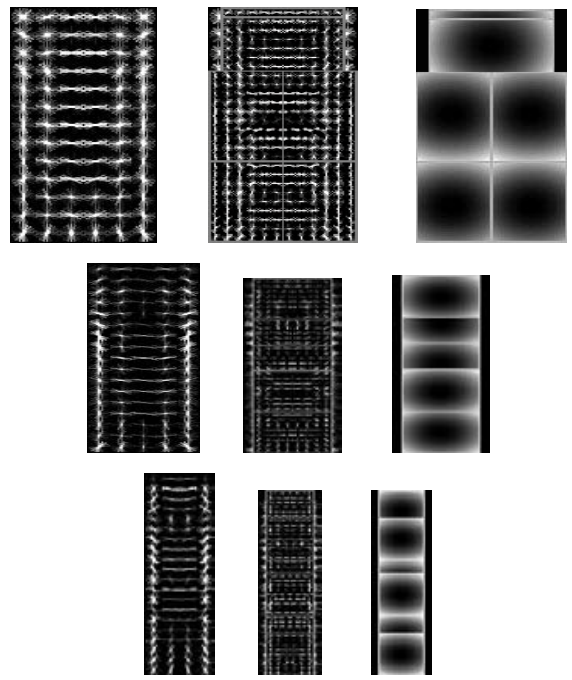

Figure 4. Visualization of spine DPM model

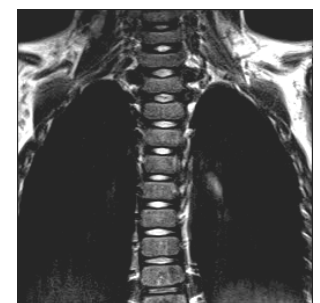

(a) Original spinal image

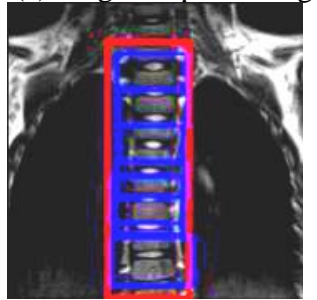

(c) Localization using DPM

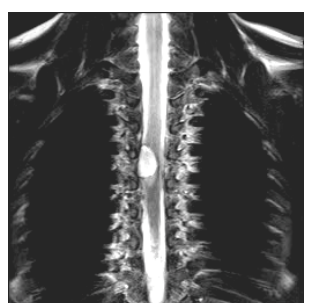

(b) Original scar image

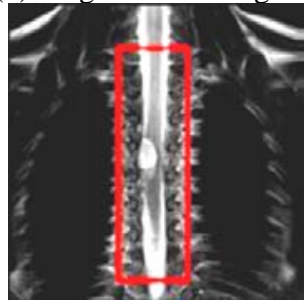

(d) Coarse ROI

Figure 5. Results of DPM detection

The location of spine can be detected successfully and the detection results as shown in Figure 5(c). Since not all slices have spinal cord, the slice index is first decided. Then scars formed at spinal cord injury are located at the central spine. Therefore, ROI is obtained according to the selected slice and neighborhood slices. The localization results are shown in Figure 5(d). 


\subsection{Precise localization of spinal cord injury}

Precise localization tries to segment SCI in all related slices for accuracy of pixel level. It includes a binarization using FCM and a growing method. MRI images have high density resolution and the density differs from tissue to tissues. Although the localization results in section 2.1 have eliminated a large amount of redundant information in image sections, there is still plenty of redundant information and undistinguishable parts in the images, such as bones, grey matters, white matters and other tissues, etc. Meanwhile, the distinction between white matters and gray matters in spinal cord is very important for the segmentation of diseased tissues. From the perspective of fuzziness, all kinds of biological tissues and organs are connected by certain degrees. As the uncertainty of the FCM algorithm has good descriptive power for images, it is suitable for segmentation problems that cannot be precisely defined in medical images. The FCM clustering algorithm uses the iterative method to obtain a clustering center with the minimum objective function, as well as the membership degree of each sample for each category [10-11].The results of division are shown in Figure 6(a).

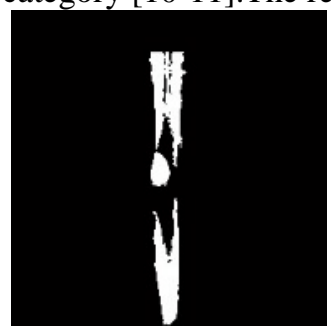

(a) FCM segmentation result

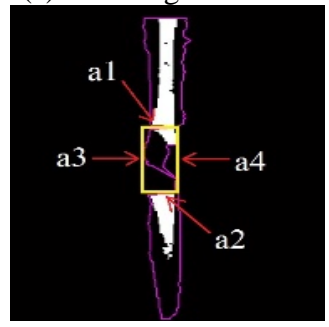

(c) Scar fine ROI

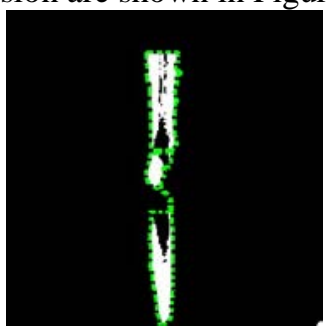

(b) Boundary detection results

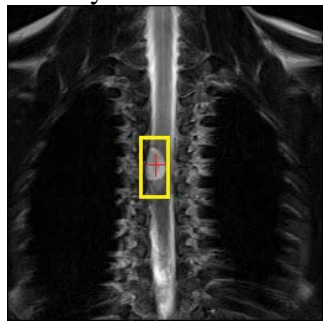

(d) Initial growing seeds

Figure 6. Precise segmentation of SCI

After binarization, this paper scans the results line by line and detects the first foreground pixel and the last foreground pixel of each line. Then it connects these pixels clockwise to form a new spinal cord boundary region. The contour of the spine is shown with dotted line in figure 6(b). Undamaged spinal cord contains complete gray matter and white matter. And the existence of the scar would result in truncation of gray matter. As there is a good distinction between gray matter and white matter information in spinal cord, this paper further narrows the detection scope of the scar based on the connectivity of gray matter. Since scars cut gray matter into two parts, the paper uses the lower edge of the upper piece as fine ROI's upper boundary $a_{1}$, the upper edge of the downmost connected domain as fine ROI's lower boundary $a_{2}$, the leftmost edge of spinal cord boundary region $\mathrm{f}_{\mathrm{n}}$ within upper and lower boundary region as fine ROI's left boundary $a_{3}$ and the rightmost boundary as fine ROI's right boundary $a_{4}$. Fine ROI is shown in figure 6(c).

Because fine ROI still contains some interference regions, centroid points are therefore extracted within the segmented ROI region according to formula 3.

$$
x_{O}=\frac{\sum_{x} \sum_{y} x \cdot I(x, y)}{\sum_{x y} I(x, y)} ; \quad y_{O}=\frac{\sum_{x} \sum_{y} y \cdot I(x, y)}{\sum_{x y} I(x, y)}
$$


Where $I(x, y)$ denotes the pixel value at position $(x, y)$ of the binarization image. Region growing method is adopted to obtain a final segmentation results from the centroid. Segmentation results are shown in Figure 7(a).

In order to improve the visual effect of SCI medical image diagnosis, 3D reconstruction was performed on the spinal cord segmentation results, using the remarkable features of VTK. In this way, doctors were allowed to acquire more accurate and intuitive visual effects in the injury site as far as possible when analyzing a patient's state of illness. The 3D reconstruction results are shown in Figure 7(b).

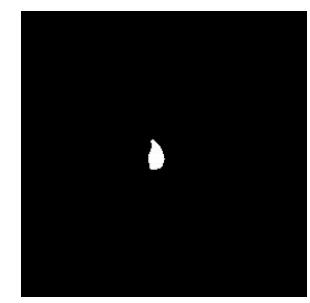

(a) Final segmentation result

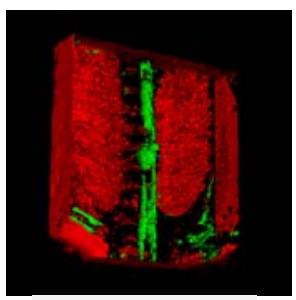

(b) $3 \mathrm{D}$ reconstruction

Figure 7. VTK 3D reconstruction

\section{Experimental results and analysis}

In order to verify results of filtering experiment objectively, this paper used image Peak Signal to Noise Ratio (PSNR) to evaluate the filtering quality of image objectively. PSNR can reflect the degree of influence of power and destructive noises on image quality. Typically, we used it as an evaluation index for the degree of image distortion. according to formula 4:

$$
P S N R=10 \times \log \left(\left(2^{n}-1\right) / M S E\right)
$$

Where $n$ stands for binary digits that a pixel in an image uses. MSE stands for mean-square error between two images. Suppose that $f(i, j)$ stands for the original image and $k(i, j)$ stands for the filtered image. Where $i=1,2, \ldots, M, j=1,2, \ldots, N, M S E$ according to formula 5 :

$$
M S E=\frac{\sum_{i=1}^{M} \sum_{j=1}^{N}[f(i, j)-k(i, j)]^{2}}{M \times N}
$$

Experimental results are shown in Table 1.

Table 1. Compare the PSNR value of different algorithms

\begin{tabular}{ccccc}
\hline PSNR/dB & $\begin{array}{c}\text { Our } \\
\text { algorithm }\end{array}$ & $\begin{array}{c}\text { Median } \\
\text { filter }\end{array}$ & $\begin{array}{c}\text { Mean } \\
\text { filter }\end{array}$ & $\begin{array}{c}\text { Gaussian } \\
\text { filter }\end{array}$ \\
\hline $\begin{array}{c}\text { Original } \\
\text { image }\end{array}$ & 20.1306 & 19.3447 & 14.4166 & 12.8659 \\
\hline
\end{tabular}

With the comparison to common smoothing filtering algorithm. This method can not only suppress current noise effectively in image, at the same time, it can keep effective edge information in the organization structure as much as possible, so as to make full use of the information.

In order to verify the proposed algorithm, a dataset of T2 weighted MRI images with SCI is formed, which includes images with SCI of 9 patients in axial or sagittal direction. Comparison with snake segmentation algorithm and traditional regional growth algorithm is also done and experimental results are shown in Figure 8. 

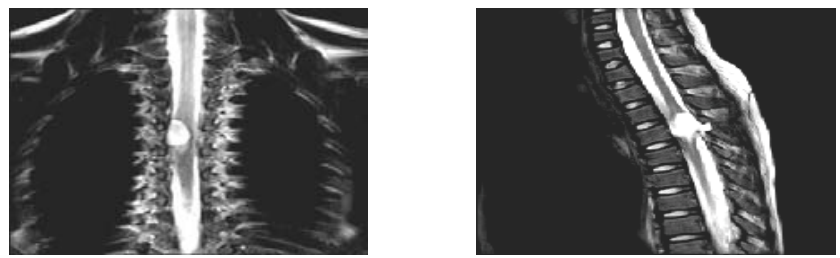

(a) Original image
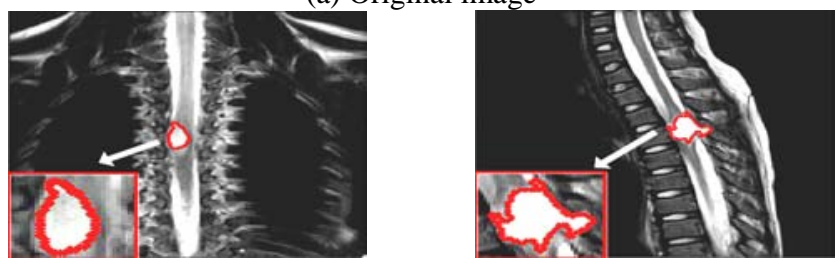

(b) The proposed approach
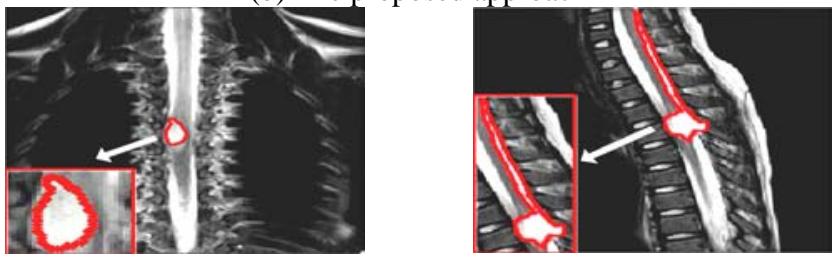

(c) Traditional region growth algorithm
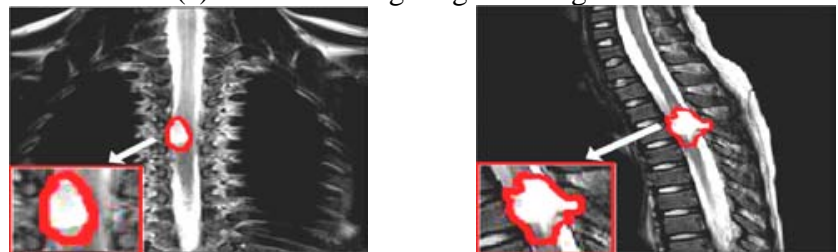

(d) Snake algorithm

Figure 8. Experience results

Through comparison, it can be seen that neither snake algorithm nor regional growth algorithm can segment the scars accurately. Moreover, both algorithms required operators to choose an initial position. If the initial position was not appropriate, the segmentation would go wrong.And the MRI images of SCI of two different patients in Figure 8 are in axial and sagittal direction respectively, so it showed the robustness of the proposed method. Figure 9 showed is a segmentation results that most experts agree. To judge whether the segmentation results of the proposed algorithm were good or bad quantitatively, fallout ratio $P_{1}$ and omission ratio $P_{2}$ statistics were conducted on scar segmentation results from the proposed algorithm and artificial segmentation results from experts. according to formula 6 :

$$
P_{1}=\frac{Q_{1}}{Q_{2}+Q_{3}} ; \quad P_{2}=\frac{Q_{3}}{Q_{2}+Q_{3}}
$$

Where $Q_{1}$ stands for the number of pixels in a non-scar area that are wrongly classified into a scar area by the proposed algorithm. $Q_{2}$ stands for the number of pixels correctly classified into a scar area by the proposed algorithm. $Q_{3}$ stands for the number of pixels in a real scar area omitted by the proposed algorithm. $Q_{4}$ stands for the number of pixels correctly classified into a non-scar area by the proposed algorithm. The statistical results of omission rate and fallout rate of sections by the proposed algorithm into a scar area are shown in Table 2 and Table 3. 


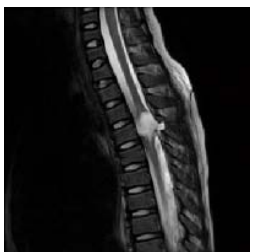

(a) Original image

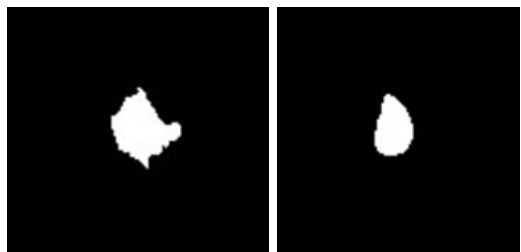

(b) Experts segmentation results

Figure 9. Results of SCI segmentation

Table 2. The fallout ratio and omission ratio of scar segmentation algorithm in thoracic spinal traumatic coronal imaging

\begin{tabular}{ccc}
\hline $\begin{array}{c}\text { The Layer of a } \\
\text { thoracic spinal } \\
\text { traumatic coronal } \\
\text { section }\end{array}$ & Fallout ratio $P_{1}$ & Omission ratio $P_{2}$ \\
\hline The $4^{\text {th }}$ Layer & 0 & 0.2107 \\
\hline The $5^{\text {th }}$ Layer & 0.0089 & 0.2026 \\
\hline The $6^{\text {th }}$ Layer & 0.0148 & 0.1751 \\
\hline
\end{tabular}

Table 3. The fallout ratio and omission ratio of scar segmentation algorithm in thoracic spinal traumatic sagittal imaging

\begin{tabular}{ccc}
\hline $\begin{array}{c}\text { The Layer of a } \\
\text { thoracic spinal } \\
\text { traumatic sagittal } \\
\text { section }\end{array}$ & Fallout ratio $P_{1}$ & Omission ratio $P_{2}$ \\
\hline The $4^{\text {th }}$ Layer & 0.0208 & 0.1810 \\
\hline The $5^{\text {th }}$ Layer & 0 & 0.2372 \\
\hline The $6^{\text {th }}$ Layer & 0.0182 & 0.1405 \\
\hline The $7^{\text {th }}$ Layer & 0.0073 & 0.1077 \\
\hline
\end{tabular}

In the segmentation experiment, we also found that there wasn't a large gap between the gray scale of some scar tissues and healthy spinal cord tissues. The gray scale of some tissues fell between healthy tissues and injured tissues. Even some scar tissues have normal spinal cord components. On the other hand, if there were normal spinal cord components in a scar tissue, in this case, it would be difficult for the algorithm to segment correctly, which led to a high omission rate of the proposed algorithm.

\section{Conclusions}

This paper aims at studying the segmentation algorithms of SCI. The repair of SCI has become a focus of study in recent medical field; however, the segmentation of medical image in traditional sense is not ideal for segmentation of SCI in terms of accuracy and convenience.

The innovation of this paper is that it segments SCI automatically. To fulfill this purpose, it adopted a coarse-to-fine strategy. This paper combined DMP detection algorithm and FCM segmentation algorithm to narrow the detection range gradually and determine the accurate range of SCI eventually.

\section{References}

1. Huang Wenbo, Yan Yang, Wang Yunji. Journal of Changchun Normal University (Natural Science), 32, 22-25(2013) 
2. Ye Chaoqun, Sun Tiansheng, Liu Zhi. Chinese Journal of Spine and Spinal Cord , 177,15718(2007).

3. Xiaoqi Lu, Jianshuai Wu, Xiaoying Ren, et al. International Journal for Light and Electron Optics, 125, 2142-2147(2014).

4. Geng-Cheng Lin, Wen-June Wang,Chung-Chia Kang, et al. Magnetic Resonance Imaging, 30, 230-246(2012).

5. Sawrav Roy, Susanta Mukhopadhyay, Manoj K. Mishra. Ultrasonics, 51, 181-189 (2011).

6. Sawrav Roy, Susanta Mukhopadhyay, Manoj K. Mishra. Pattern Recognition, 48, 22542268(2015).

7. P. Felzenszwalb, D.McAllester, D.Ramaman. Proceedings of the IEEE CVPR ,1-8 (2008).

8. P. Felzenszwalb, R. Girshick, D. McAllester, D. Ramanan. IEEE Transactions on Pattern Analysis and Machine Intelligence, 32, 1627-1645 (2010).

9. Gerard Pons, Robert Martí,Sergi Ganau, et al. Ultrasound in Medicine \& Biology, 40, 22522264(2014).

10. Kaur, Prabhjot, Soni, A.K.,et al.Pattern Recognition Letters, 34, 163- 175 (2012).

11. Liu Y, Xiao K, Liang A, et al. International Conference on Hybrid Artificial Intelligent Systems, 221-230(2012).

12. Pengchong Jin, C. A. Bouman, and K. D. Sauer , IEEE Transactions on Computational Imaging, 1, 200-216(2015).

13. Pengchong Jin, Dong Hye Ye, and C. A. Bouman , ICIP , (2015).

14. Pengchong Jin, E. Haneda, C. A. Bouman, and K. D. Sauer, 2-nd CT meeting, 297-300(2012).

15. De, Leener B, S. Kadoury, and J. Cohen-Adad. Neuroimage , 98, 69-71(2014). 